\title{
Micromechanical Modeling of Deployment and Shape Recovery of Thin-walled Viscoelastic Composite Space Structures
}

\author{
Kawai Kwok* and Sergio Pellegrino ${ }^{\dagger}$ \\ California Institute of Technology, Pasadena, CA 91125
}

\begin{abstract}
The first part of the paper presents an experimental study of the deployment and shape recovery of composite tape-springs after stowage at an elevated temperature. It is found that tape-springs deploy quickly and with a slight overshoot, but complete recovery takes place asymptotically over time. Stowage has the effect of slowing down both the shortterm deployment and long-term shape recovery. The second part of the paper presents a micromechanical finite element homogenization scheme to determine the effective viscoelastic properties of woven composite laminas. This solution scheme is employed in numerical simulations of deployment and shape recovery of composite tape-springs. The proposed micromechanical model predicts both the short-term deployment and long-term shape recovery response with close agreement to the experimental measurements.
\end{abstract}

\section{Introduction and Background}

Deployable structures made of thin composite materials present a promising solution for spacecraft applications because of their high thermal stability and specific stiffness. Notable examples of composite deployable structures include the Springback Antennas on the Mobile Satellite System (MSAT) ${ }^{1}$ and the Flattenable Foldable Tubes, Fig. 1, forming the Mars Advanced Radar for Subsurface and Ionospheric Sounding (MARSIS) antenna on the Mars Express Spacecraft. ${ }^{2}$

A common issue in designing composite deployable structures is the inherent creep behavior of the polymer matrix, which often limits the amount of deployment force and the shape precision that can be achieved. As deployable structures are routinely stowed for extended periods and subject to varying temperature environments, realistic predictions on, for instance, the loss in deployment force during stowage and the time required for a complete shape recovery are required for robust designs. A detail material model that accurately computes viscoelastic composite properties can effectively guide the design process of composite deployable structures.

Viscoelastic behavior of deployable structures has started to be addressed only recently. While experimental results on recovery time and vibration characteristics of composite tubes after stowage have been reported, ${ }^{3,4}$ a general method of analysis is lacking. A more fundamental study of viscoelastic deployable structures was undertaken by Kwok and Pellegrino, who employed a linear viscoelastic constitutive model to study the folding, stowage, and deployment of beams ${ }^{5}$ and tape-springs ${ }^{6}$ as a time-continuous event. These previous studies were limited to homogeneous viscoelastic materials.

Micromechanical material modeling of viscoelastic composites is more well studied. Many analytical models have been developed for determining viscoelastic moduli of unidirectional composites from constituent fiber and matrix properties. The underlying approach is to extend existing micromechanical models for elastic composites to viscoelastic composites by exploiting the elastic-viscoelastic correspondence principle. ${ }^{7,8}$ Viscoelastic moduli have also been obtained numerically through direct finite element analysis ${ }^{9}$ assuming a uniform distribution of fibers. For woven composites, Govindarajan et al. ${ }^{10}$ and Shrotriya and Sottos ${ }^{11}$ proposed analytical models based on simplified weave geometries, but the flexural deformation of the fiber

\footnotetext{
*Ph.D. Candidate, Graduate Aerospace Laboratories, 1200 E. California Blvd. MC 205-45. kwk5@caltech.edu

${ }^{\dagger}$ Joyce and Kent Kresa Professor of Aeronautics and Professor of Civil Engineering, Graduate Aerospace Laboratories, 1200 E. California Blvd. MC 301-46. AIAA Fellow. sergiop@caltech.edu
} 
tows was not accounted for and was believed to be the source of significant discrepancy between predicted and measured viscoelastic responses. Predictions were improved when two-dimensional ${ }^{11}$ and three- dimensional $^{12}$ finite element based methods were utilized to incorporate the influence of weave geometry, but still did not match the experimental results.

The present investigation bridges the gap between existing micromechanical modeling techniques for viscoelastic composite materials and global analysis of large deployable structures. We present a detailed study of a particular structure that poses the general challenges typical of thin-walled deployable structures with viscoelastic properties and then demonstrate the modeling techniques for predicting its response. The particular structure selected is a composite tape-spring made of epoxy matrix reinforced with plain weave carbon fabric. Tape-springs are thin shells with curved section, typically of uniform curvature and subtending an angle smaller than $180^{\circ}$, Fig. 2. This structural form can be folded by forming a local buckle and is therefore routinely employed as self-deployable hinges on spacecrafts. In the current study, carefully controlled experiments on stowage, deployment, and shape recovery of composite tape-springs are presented along with numerical predictions using a finite element based micromechanical model for thin composites consisting of linear viscoelastic matrix and linear elastic fibers.

The paper is arranged as follows. Section II describes the experimental procedures and presents the results of deployment and shape recovery experiments. Section III presents the the finite-element based homogenization scheme for viscoelastic behavior of thin composites. The model is employed in Section IV to simulate the viscoelastic response of tape-springs. The predicted deployment and shape recovery response are compared with measured responses in Section V. Section VI discusses the findings of the study and concludes the paper.

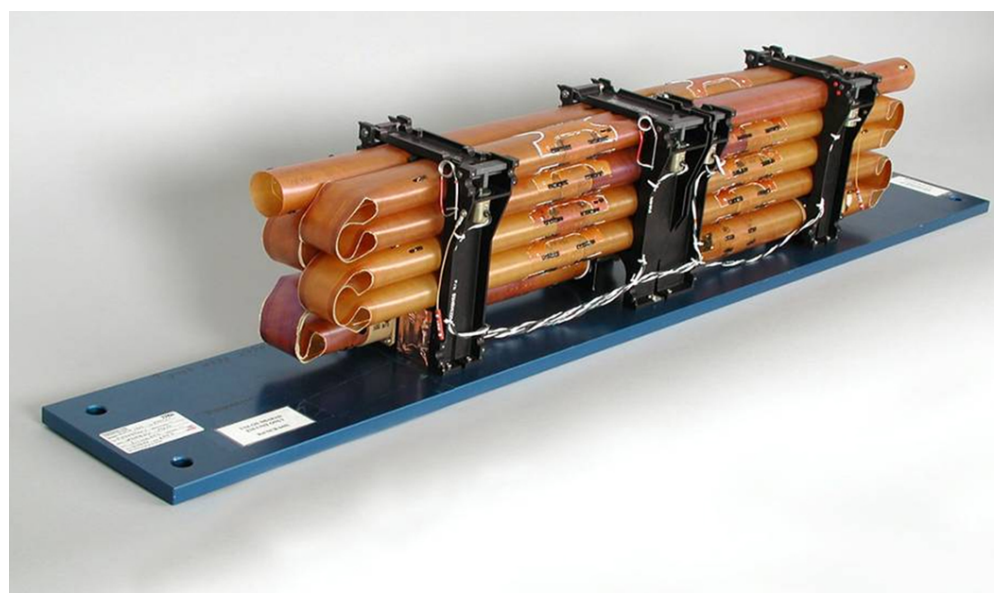

Figure 1: Flattenable foldable tubes used on MARSIS (Astro Aerospace).

\section{Deployment and Shape Recovery Experiments}

To assess the deployment and shape recovery behavior after stowage, experiments were carried out on composite tape-springs with a diameter of $38 \mathrm{~mm}$, a thickness of $0.125 \mathrm{~mm}$, a length of $596 \mathrm{~mm}$, and an areal density of $131.2 \mathrm{~g} / \mathrm{m}^{2}$. The tape-springs were made of \pm 45 plain-weave fabric with $1 \mathrm{k}$ tows of T300 carbon fibers impregnated with PMT-F4 epoxy resin. They were fabricated by laying the resin-impregnated fabric on a steel mandrel, wrapping the assembly in release films, and cured under vacuum for $2 \mathrm{~h}$ at $120^{\circ} \mathrm{C}$ and $400 \mathrm{kPa}$.

An important feature of viscoelastic behavior in polymers is the temperature dependence of the relaxation time. Polymers behave almost elastically at temperatures well below the glass transition temperature $T_{g}$. The change of material response over time accelerates as the temperature moves towards $T_{g}$. Since the glass transition temperature for PMT-F4 is $133^{\circ} \mathrm{C}$, an unrealistically long stowage time would be required before noticeable viscoelastic response can be observed at room temperature. Tape-springs were therefore stowed at a higher temperature in the experiments to increase the rate of relaxation.

Each test consisted of stowing the tape-spring for a given length of time at a specified temperature, 


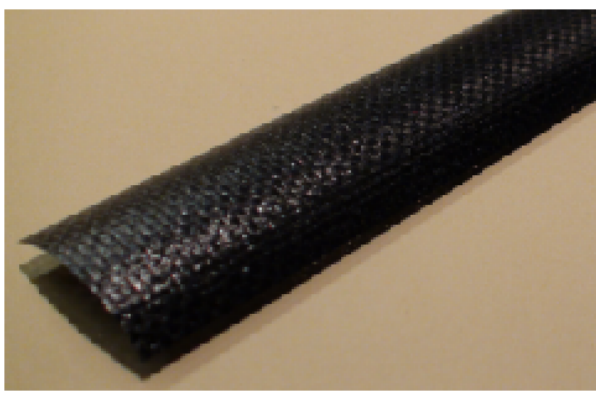

(a)

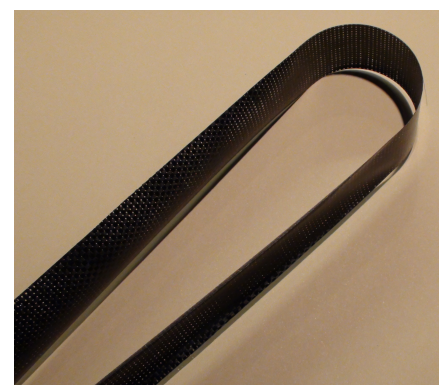

(b)

Figure 2: Composite tape-spring: (a) deployed and (b) folded.

deploying it, and measuring the shape change over time after deployment. Experiments were performed inside a thermal chamber that stabilizes the temperature of the specimens within $0.1^{\circ} \mathrm{C}$ over the test period. The test specimen was clamped on the bottom and positioned vertically on a granite table to reduce background vibration. Thermocouples were placed at three different locations in the vicinity of the specimen to monitor the temperature uniformity, which can be controlled within $\pm 0.5^{\circ} \mathrm{C}$. In previous studies, it was discovered that the behavior of viscoelastic tape-springs is characterized by a short-term deployment phase followed by a long-term recovery phase. Tape-springs undergo large displacements within a short period during deployment and exhibit infinitesimal deformations over extended periods during shape recovery. Two displacement measurement schemes were therefore employed to probe the behavior under such drastically different length and time scales. The deployed angle was extracted from images taken using a high resolution camcorder with a frame rate of $30 \mathrm{fps}$. Full field displacements of the fold region in the tape-spring were measured using a three dimensional digital image correlation system during the recovery phase. The system consists of two Point Grey Research CCD cameras with a resolution of 2448 x 2048 and a pixel size of $3.45 \mu m \times$ $3.45 \mu \mathrm{m}$. The cameras were positioned to capture a series of images of the inner surface of the tape-spring throughout the test. The experimental configuration is shown in Fig. 3.

The test procedure proceeded as follows. The chamber was first heated and stabilized at $60^{\circ} \mathrm{C}$. The chamber was then opened for manually stowing the tape-spring to an angle of $90^{\circ}$. To minimize the disturbance of the thermal environment, the chamber was kept opened for only a short time. The specimen was stowed for a period of $8 \mathrm{~h}$ and then deployed. Displacements were measured continuously over time from the instant of deployment to $8 \mathrm{~h}$ after deployment. The same experiment was repeated at $23^{\circ} \mathrm{C}$ as a reference for comparison.

As noted previously, the behavior of tape-springs after stowage can be divided into two stages, namely deployment and recovery. Fig. 4 plots the measured deployed angle over time for both temperatures and Fig. 5 shows a series of intermediate shapes of the tape-spring during deployment at $60^{\circ} \mathrm{C}$. In both cases, the tape-spring passes through the undeformed configuration and overshoots by a small amount, after which the deployed angle gradually tends towards zero. It is noted in Fig. 5 that the fold location is constant throughout deployment. The same behavior was observed in polymer tape-springs ${ }^{6}$ and is different from that of metallic tape-springs in which deployment is accompanied by the fold traveling towards the fixed end. ${ }^{13}$ The effect of stowage at two different temperatures is apparent in Fig. 4 . At $23^{\circ} \mathrm{C}$, the maximum overshoot occurred at $0.4 \mathrm{~s}$ with a magnitude of $-1.4^{\circ}$. At $60^{\circ} \mathrm{C}$, the overshoot was delayed to $0.65 \mathrm{~s}$ with a larger angle of $-2.5^{\circ}$.

Fig. 6 shows the measured out-of-plane displacement field in the fold region of the tape-spring superimposed on images of the specimen over a longer time span at $60^{\circ} \mathrm{C}$. The displacement field measurements were obtained from analyzing images of the deformed specimens with the Vic-3D digital image correlation software. The principle of analysis is the following. A reference image is first chosen and divided into small square regions. The software then matches these regions between any subsequent image and the reference image based on pattern recognition algorithms and determine the movements of the regions between the two 
images. The result is then a displacement field for each image relative to the reference. To illustrate the small movements during recovery, the image taken at the instant of maximum overshoot was chosen to be the reference. As shown in Fig. 6, an out-of-plane displacement of about $3 \mathrm{~mm}$ was recorded over $5743 \mathrm{~s}$ $(1.6 \mathrm{~h})$. This indicates that the cross-sectional shape of the tape-spring was slowly changing over a long period after deployment, even though the tape-spring had nearly reached the undeformed configuration in about $1 \mathrm{~s}$. If the tape-spring had been made of a time-independent material, the cross section would have fully recovered and arrived at the fully deployed configuration in a few seconds.

The presented experimental results demonstrate that stowage has the effect of slowing down deployment and shape recovery in viscoelastic composite structures.

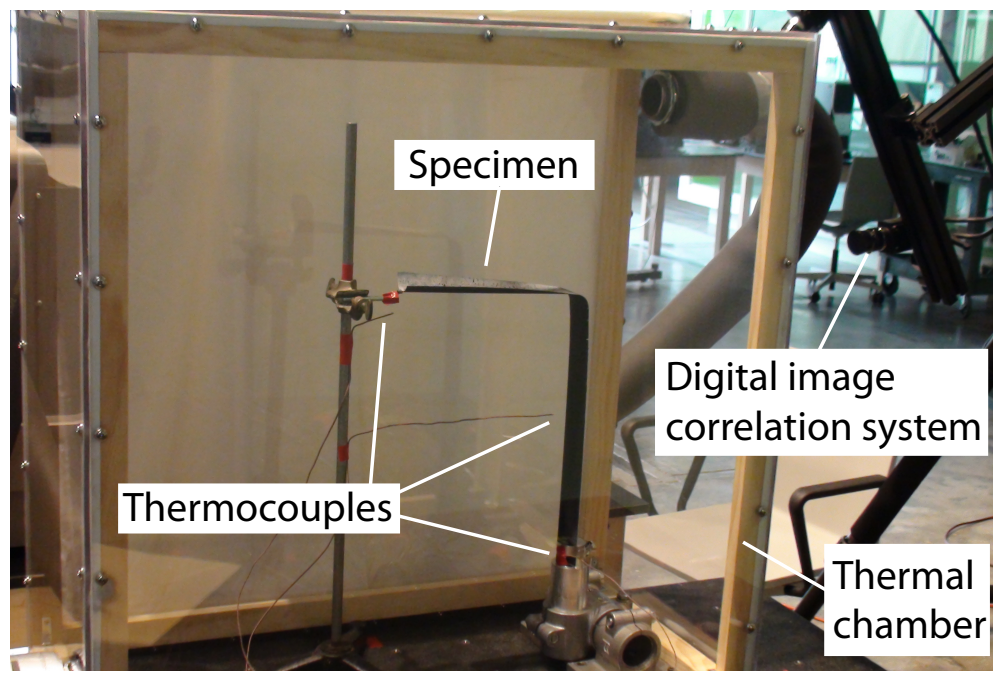

Figure 3: Experimental configuration.

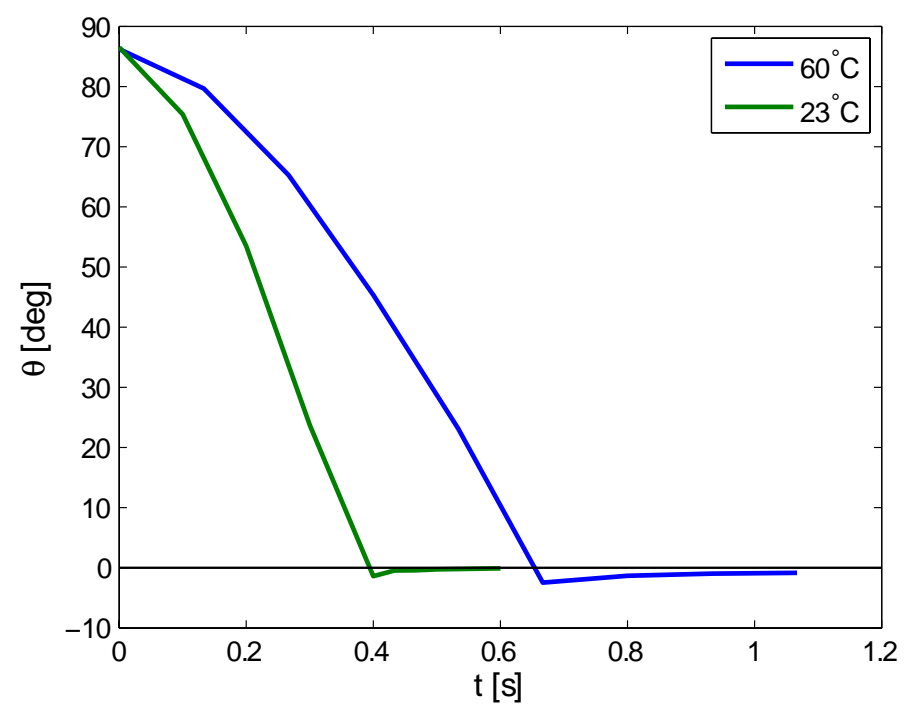

Figure 4: Measured deployed angle vs. time. 


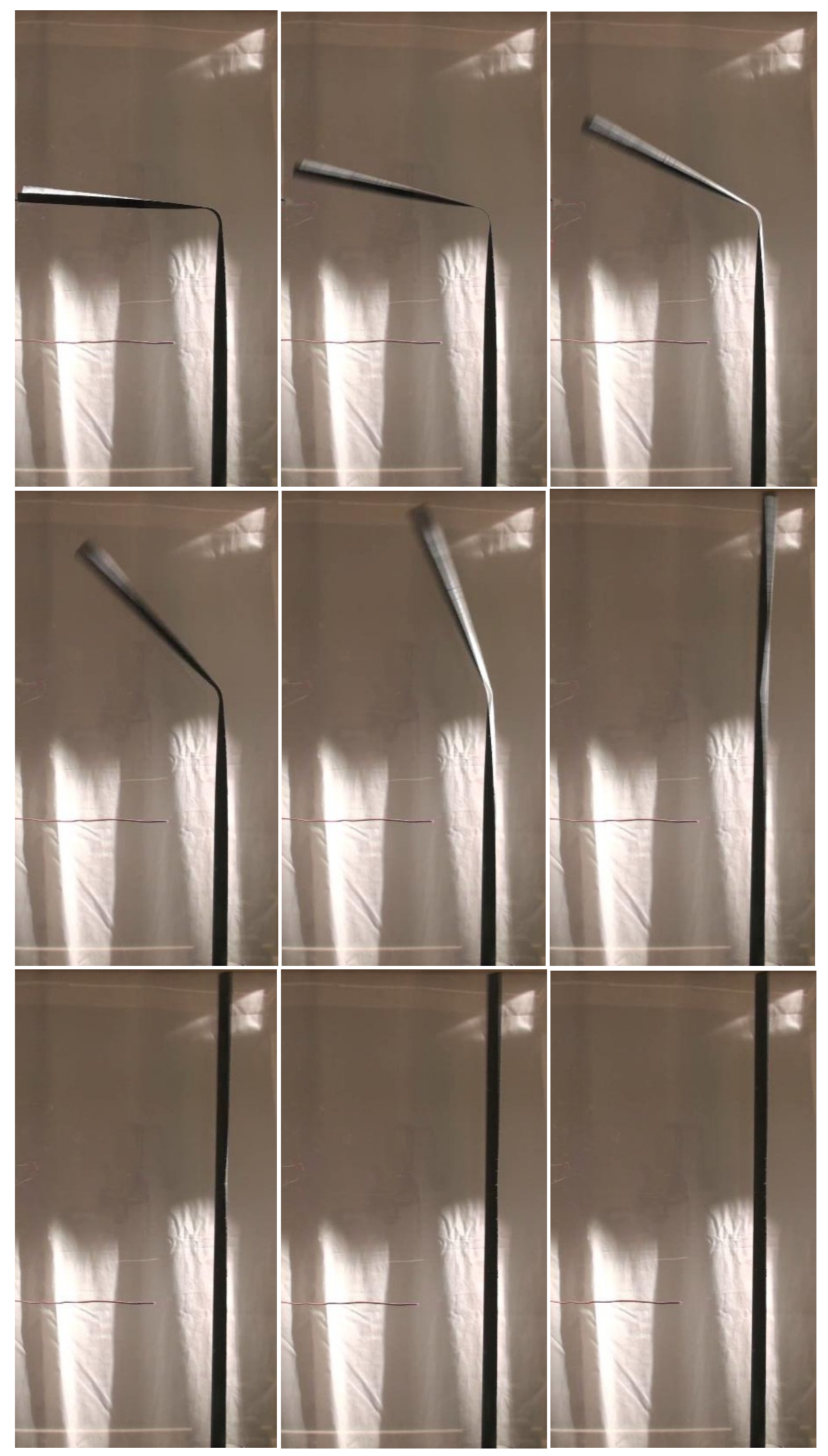

Figure 5: Snapshots at $0 \mathrm{~s}, 0.13 \mathrm{~s}, 0.26 \mathrm{~s}, 0.39 \mathrm{~s}, 0.52 \mathrm{~s}, 0.65 \mathrm{~s}, 0.78 \mathrm{~s}, 0.91 \mathrm{~s}$, and $1.04 \mathrm{~s}$ of deployment sequence. 


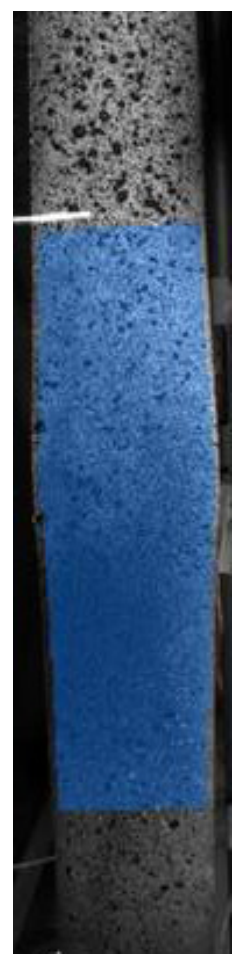

(a)

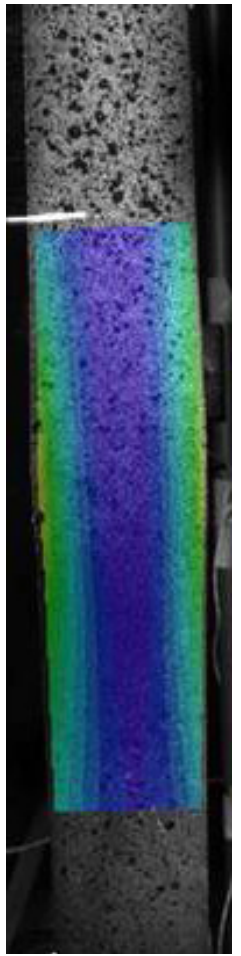

(b)

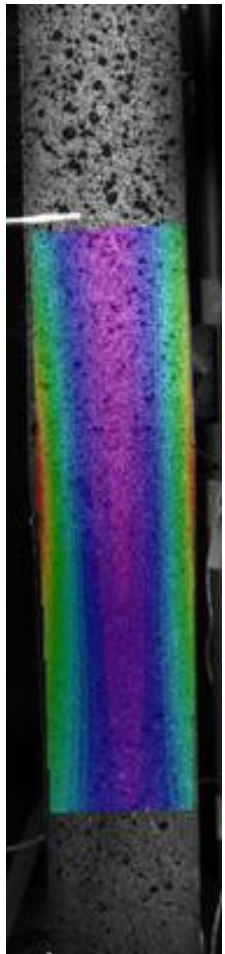

(c)

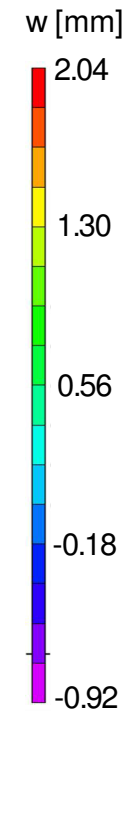

0.56

0.18

0.92

Figure 6: Measured out-of-plane displacements of the fold region relative to the configuration of maximum overshoot: (a) at overshoot (reference), (b) after $50 \mathrm{~s}$, and (c) after $5743 \mathrm{~s}$. 


\section{Micromechanical Modeling of Viscoelastic Composites}

The effective viscoelastic response of a woven composite lamina consisting of elastic carbon fibers embedded in viscoelastic epoxy matrix depends on factors such as the matrix relaxation moduli, weave geometry and tow sizes. To model material and geometric effects at lower length scales, a finite element homogenization approach is proposed in the present study. In particular, homogenization of the viscoelastic properties is carried out both at the tow level and at the lamina level using the properties of its constituents.

\section{A. Constituent Properties}

The epoxy matrix is treated as an isotropic viscoelastic solid and its time and temperature dependent mechanical properties are modeled using a Prony series. The Prony series representation of the relaxation modulus is written as

$$
E(t)=E_{\infty}+\sum_{i=1}^{n} E_{i} e^{-\left(t / \rho_{i}\right)},
$$

where $t$ is time, $E_{\infty}$ is the long term modulus, $E_{i}$ are the Prony coefficients, and $\rho_{i}$ are the relaxation times.

The effects of temperature on the material behavior can be treated in the same manner through the time-temperature superposition principle, ${ }^{14}$ which states that the modulus at temperature $T$ and time $t$ is the same as the modulus at a reference temperature $T_{0}$ and at a reduced time $t^{\prime}$,

$$
E(t, T)=E\left(t^{\prime}, T_{0}\right), \quad t^{\prime}=\frac{t}{a_{T}(T)},
$$

where $a_{T}(T)$ is the temperature shift factor. Thus, one can relate the viscoelastic behavior at one temperature to that at another temperature by a shift in the time scale. When the temperature varies with time, the reduced time is obtained by integration,

$$
t^{\prime}(t)=\int_{0}^{t} \frac{d \tau}{a_{T}(T(\tau))}
$$

Based on this principle, a master curve can be constructed at an arbitrary reference temperature by shifting the relaxation moduli at any other temperatures to the reference temperature. On a log-log plot of relaxation versus time, this is equivalent to a horizontal shift with a distance of $\log a_{T}(T)$. The resulting master curve is a plot of relaxation modulus on the reduced time scale and describes both the time and temperature dependence of the material behavior.

A commonly used relation for the temperature shift factor of polymers is the Williams-Landel-Ferry (WLF) equation, ${ }^{15}$

$$
\log a_{T}=-\frac{C_{1}\left(T-T_{0}\right)}{C_{2}+\left(T-T_{0}\right)},
$$

in which $C_{1}$ and $C_{2}$ are material constants that depend on the particular polymer and the logarithm is of base ten.

The master curve for PMT-F4 epoxy matrix is obtained via creep tests and is shown in Fig. 7. The corresponding parameters that describe the Prony series representation of the relaxation modulus are summarized in Table 1. The Poisson's ratio of the matrix is assumed to be constant over time and is determined to be 0.33 . The material constants $C_{1}$ and $C_{2}$ were found by fitting the temperature shift data to the WLF equation, Eq. (4), and the values obtained for the reference temperature $T_{0}=40^{\circ} \mathrm{C}$ were

$$
C_{1}=28.3816 \quad \text { and } \quad C_{2}=93.291
$$

Carbon fibers are regarded as transversely isotropic elastic solid. The mechanical properties of T300 fibers are listed in Table 2 . 


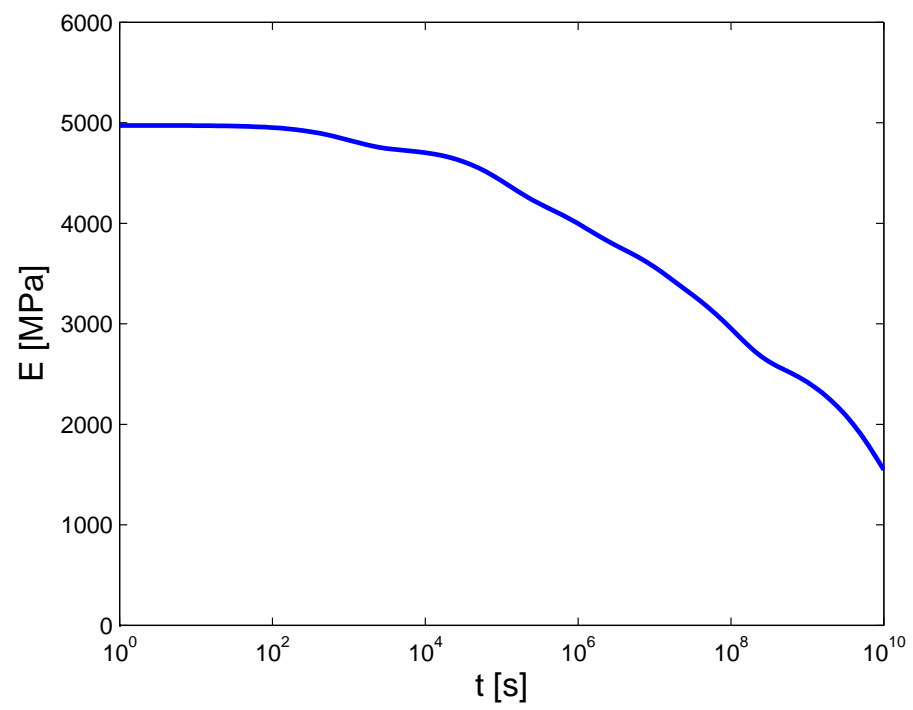

Figure 7: Master curve of PMT-F4 at a reference temperature of $\mathrm{T}=40^{\circ} \mathrm{C}$.

\begin{tabular}{ccc}
\hline$i$ & $E_{i}[\mathrm{MPa}]$ & $\rho_{i}[\mathrm{~s}]$ \\
\hline$\infty$ & 1000 & - \\
1 & 224.1 & $1.0 e+3$ \\
2 & 450.8 & $1.0 e+5$ \\
3 & 406.1 & $1.0 e+6$ \\
4 & 392.7 & $1.0 e+7$ \\
5 & 810.4 & $1.0 e+8$ \\
6 & 203.7 & $1.0 e+9$ \\
7 & 1486.0 & $1.0 e+10$ \\
\hline
\end{tabular}

Table 1: Relaxation times and relaxation coefficients for PMT-F4.

\begin{tabular}{cc}
\hline Properties & Value \\
\hline$E_{1}[M P a]$ & 233,000 \\
$E_{2}[M P a]$ & 23,100 \\
$G_{12}[M P a]$ & 8,963 \\
$\nu_{12}$ & 0.2 \\
$\nu_{23}$ & 0.4 \\
\hline
\end{tabular}

Table 2: Elastic properties of T300 carbon fibers. 


\section{B. Viscoelastic Behavior of Tows}

The viscoelastic properties of a straight and transversely isotropic fiber tow were determined from the constituent properties by direct finite element analysis of a unit cell. The cross section of a representative tow is shown in Fig. 8, from which the fiber diameter and volume fraction were determined to be $d_{f}=7.0 \mu \mathrm{m}$ and $v_{f}=0.64$ respectively. The unit cell model is composed of a single fiber surrounded by matrix. This implicitly assumes square array fiber arrangement. Hexagonal array and random array fiber arrangements were also implemented, but the difference between these configurations was found to be insignificant at this volume fraction.

The particular finite element model shown in Fig. 9 is a cube with edge length of $\ell=7.7 \mu \mathrm{m}$ and is composed of 168008 -node brick and 800 6-node triangular prism elements. The material properties defined in Sec. A were assigned to the fiber and matrix elements accordingly. Periodic displacement boundary conditions are enforced between each pair of opposite boundary faces of the unit cell through constraint equations in terms of normal and shear strains of a homogeneous continuum solid:

$$
\begin{aligned}
& u\left(\frac{\ell}{2}, y, z\right)-u\left(-\frac{\ell}{2}, y, z\right)=\epsilon_{x x} \ell \\
& v\left(\frac{\ell}{2}, y, z\right)-v\left(-\frac{\ell}{2}, y, z\right)=\epsilon_{x y} \ell \\
& w\left(\frac{\ell}{2}, y, z\right)-w\left(-\frac{\ell}{2}, y, z\right)=\epsilon_{x z} \ell \\
& u\left(x, \frac{\ell}{2}, z\right)-u\left(x,-\frac{\ell}{2}, z\right)=\epsilon_{y x} \ell \\
& v\left(x, \frac{\ell}{2}, z\right)-v\left(x,-\frac{\ell}{2}, z\right)=\epsilon_{y y} \ell \\
& w\left(x, \frac{\ell}{2}, z\right)-w\left(x,-\frac{\ell}{2}, z\right)=\epsilon_{y z} \ell \\
& u\left(x, y, \frac{\ell}{2}\right)-u\left(x, y,-\frac{\ell}{2}\right)=\epsilon_{z x} \ell \\
& v\left(x, y, \frac{\ell}{2}\right)-v\left(x, y,-\frac{\ell}{2}\right)=\epsilon_{z y} \ell \\
& w\left(x, y, \frac{\ell}{2}\right)-w\left(x, y,-\frac{\ell}{2}\right)=\epsilon_{z z} \ell
\end{aligned}
$$

where $u, v$, and $w$ denote displacements in the $x, y$, and $z$ directions respectively, and $\epsilon$ denotes strain.

The constitutive equation for a homogenized transversely isotropic solid with fibers aligned with the 1 -axis is written as

$$
[\sigma(t)]=\int_{0}^{t}([C(t-\tau)][\dot{\epsilon}(\tau)]) d \tau
$$

where $C(t)$ is written as

$$
C(t)=\left[\begin{array}{cccccc}
C_{11}(t) & C_{12}(t) & C_{12}(t) & 0 & 0 & 0 \\
C_{12}(t) & C_{22}(t) & C_{23}(t) & 0 & 0 & 0 \\
C_{12}(t) & C_{23}(t) & C_{22}(t) & 0 & 0 & 0 \\
0 & 0 & 0 & C_{44}(t) & 0 & 0 \\
0 & 0 & 0 & 0 & C_{55}(t) & 0 \\
0 & 0 & 0 & 0 & 0 & C_{55}(t)
\end{array}\right] .
$$

Only four separate analyses are needed to compute the independent time-varying functions in $C(t)$ because each analysis provides more than one function and the homogenized solid has a plane of isotropy. The analyses were carried out in Abaqus/Standard. In each analysis, a unit amplitude of one of the six strain variables in the form of a step function was applied to the unit cell and the corresponding time-dependent stresses were obtained. Each entry in the $C(t)$ matrix was modeled by a Prony series having the same relaxation times as the matrix and was computed by invoking the virtual work principle. The computed results are plotted with respect to time in Fig. 10. 


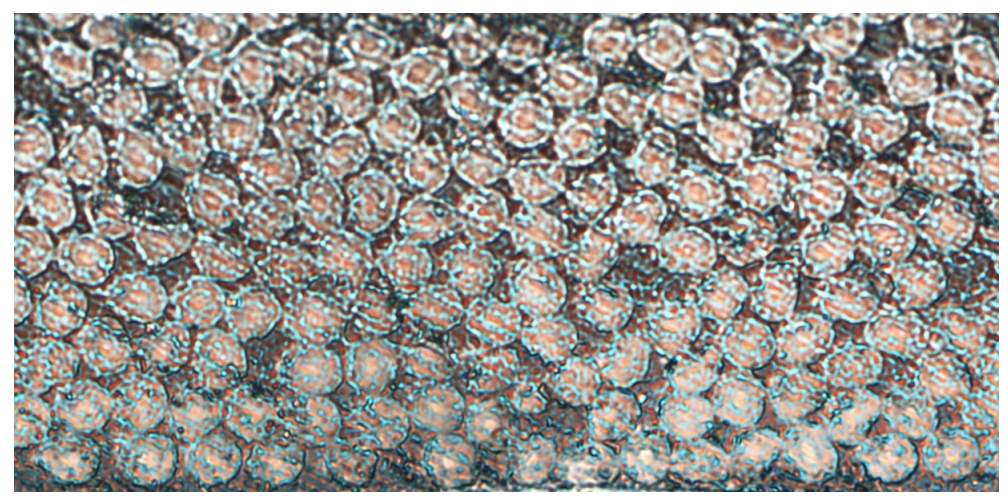

Figure 8: Cross section of a tow.

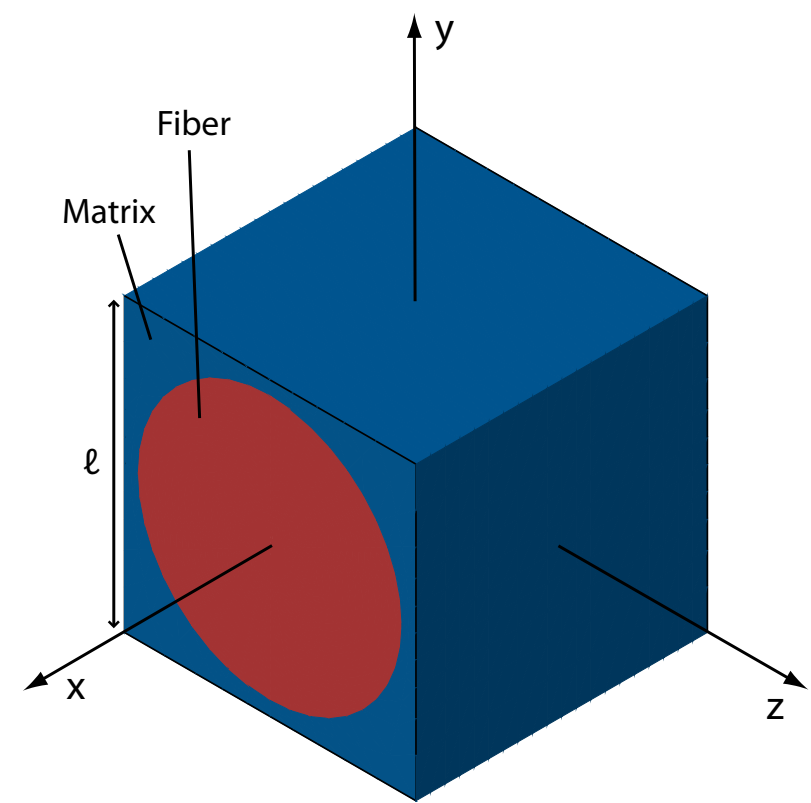

Figure 9: Finite element model for a unidirectional fiber tow unit cell. 


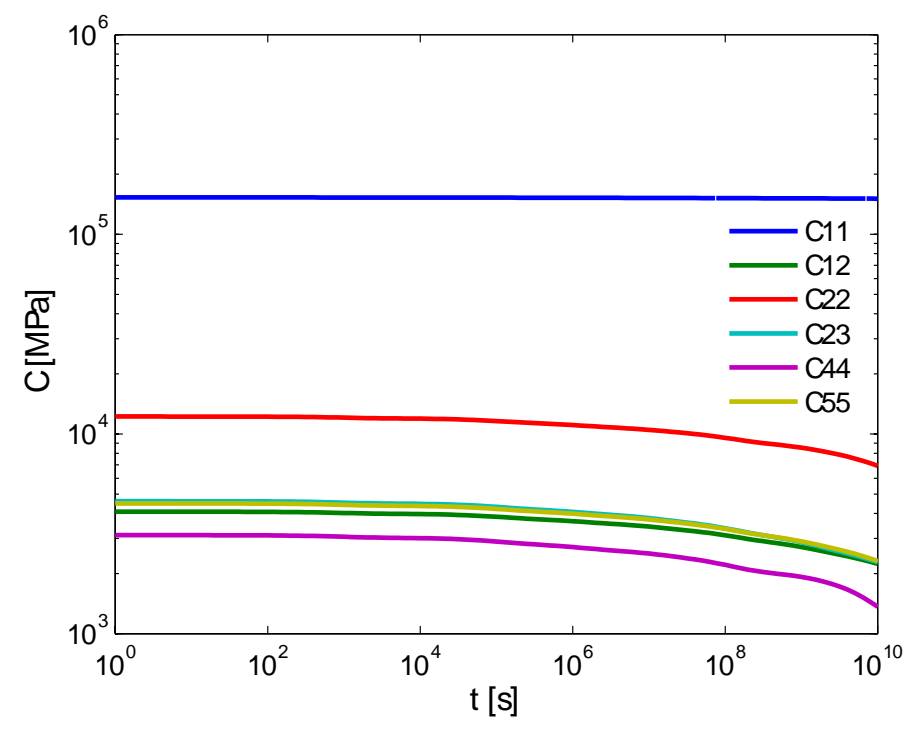

Figure 10: Viscoelastic properties of a unidirectional fiber tow.

\section{Viscoelastic Properties of Laminas}

A plain-weave composite lamina is modeled as a Kirchhoff plate consisting of viscoelastic tows and matrix. The cross section geometry of the lamina is shown in Fig. 11. It has a weave length of $L=3.5 \mathrm{~mm}$, a maximum tow thickness of $h=0.063 \mathrm{~mm}$, a tow width of $w=1.05 \mathrm{~mm}$, and an overall fiber volume fraction of $\nu_{f}=0.52$. The lamina unit cell model is composed of four tows defined by sinusoidal functions and pure matrix that fills the gaps between the tows, as shown in Fig. 12. Each tow is made of 960 brick elements and the matrix is made up of 1920 brick and 640 triangular prism elements. The material properties for the tows were defined by the relaxation moduli obtained in Sec. B through a user defined material subroutine (UMAT) in Abaqus/Standard. To impose the Kirchhoff constraint, nodes lying on the boundary faces of the unit cell are tied to nodes in the mid-plane through rigid connections. The displacements and rotations of each mid-plane node on one face are paired to the corresponding node on the opposite face by constraint equations that impose periodic boundary conditions in terms of mid-plane strains and out-of-plane curvatures of a homogenized Kirchhoff plate. The kinematic relations are

$$
\begin{gathered}
\epsilon_{x}=\frac{\partial u}{\partial x} \\
\epsilon_{y}=\frac{\partial v}{\partial y} \\
\gamma_{x y}=\frac{\partial u}{\partial y}+\frac{\partial v}{\partial x} \\
\kappa_{x}=-\frac{\partial^{2} w}{\partial x^{2}} \\
\kappa_{y}=-\frac{\partial^{2} w}{\partial y^{2}} \\
\kappa_{x y}=-2 \frac{\partial^{2} w}{\partial x \partial y} .
\end{gathered}
$$

The displacement constraints are written as

$$
u\left(\frac{L}{2}, y\right)-u\left(-\frac{L}{2}, y\right)=\epsilon_{x} L
$$




$$
\begin{gathered}
v\left(\frac{L}{2}, y\right)-v\left(-\frac{L}{2}, y\right)=\frac{1}{2} \gamma_{x y} L \\
w\left(\frac{L}{2}, y\right)-w\left(-\frac{L}{2}, y\right)=-\frac{1}{2} \kappa_{x y} y L \\
\theta_{x}\left(\frac{L}{2}, y\right)-\theta_{x}\left(-\frac{L}{2}, y\right)=-\frac{1}{2} \kappa_{x y} L \\
\theta_{y}\left(\frac{L}{2}, y\right)-\theta_{y}\left(-\frac{L}{2}, y\right)=\kappa_{x} L \\
\theta_{z}\left(\frac{L}{2}, y\right)-\theta_{z}\left(-\frac{L}{2}, y\right)=0 \\
u\left(x, \frac{L}{2}\right)-u\left(x,-\frac{L}{2}\right)=\frac{1}{2} \gamma_{x y} L \\
v\left(x, \frac{L}{2}\right)-v\left(x,-\frac{L}{2}\right)=\epsilon_{y} L \\
w\left(x, \frac{L}{2}\right)-w\left(x,-\frac{L}{2}\right)=-\frac{1}{2} \kappa_{x y} x L \\
\theta_{x}\left(x, \frac{L}{2}\right)-\theta_{x}\left(x,-\frac{L}{2}\right)=-\kappa_{y} L \\
\theta_{y}\left(x, \frac{L}{2}\right)-\theta_{y}\left(x,-\frac{L}{2}\right)=\frac{1}{2} \kappa_{x y} L \\
\theta_{z}\left(x, \frac{L}{2}\right)-\theta_{z}\left(x,-\frac{L}{2}\right)=0,
\end{gathered}
$$

where $\theta$ are rotations and the subscripts denote the rotation directions.

The constitutive relationship for the homogenized plate is written as

$$
\begin{aligned}
& {[N(t)]=\int_{0}^{t}([A(t-\tau)][\dot{\epsilon}(\tau)]+[B(t-\tau)][\dot{\kappa}(\tau)]) d \tau,} \\
& {[M(t)]=\int_{0}^{t}([B(t-\tau)][\dot{\epsilon}(\tau)]+[D(t-\tau)][\dot{\kappa}(\tau)]) d \tau,}
\end{aligned}
$$

where the matrices $[N]$ and $[M]$ denote force and moment resultants; $[A],[B]$ and $[D]$ are the in-plane stiffness, stretching-bending coupling, and bending stiffness matrices. For a plain-weave lamina, the $A B D$ stiffness matrix is written as

$$
A B D(t)=\left[\begin{array}{ccc|ccc}
A_{11}(t) & A_{12}(t) & 0 & 0 & 0 & 0 \\
A_{12}(t) & A_{11}(t) & 0 & 0 & 0 & 0 \\
0 & 0 & A_{66}(t) & 0 & 0 & 0 \\
\hline 0 & 0 & 0 & D_{11}(t) & D_{12}(t) & 0 \\
0 & 0 & 0 & D_{12}(t) & D_{11}(t) & 0 \\
0 & 0 & 0 & 0 & 0 & D_{66}(t)
\end{array}\right] .
$$

Similar to the homogenization procedure conducted for the fiber tows, each entry in the $A B D(t)$ matrix was modeled by a Prony series having the same relaxation times as the matrix. Six separate analyses, each corresponding to a unit amplitude of the six deformation variables, were carried out in Abaqus/Standard to determine all the entries. The computed results are plotted with respect to time in Fig. 13 and Fig. 14.

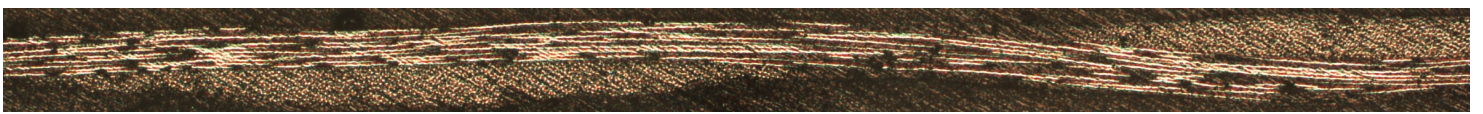

Figure 11: Cross section geometry of a plain-weave lamina. 


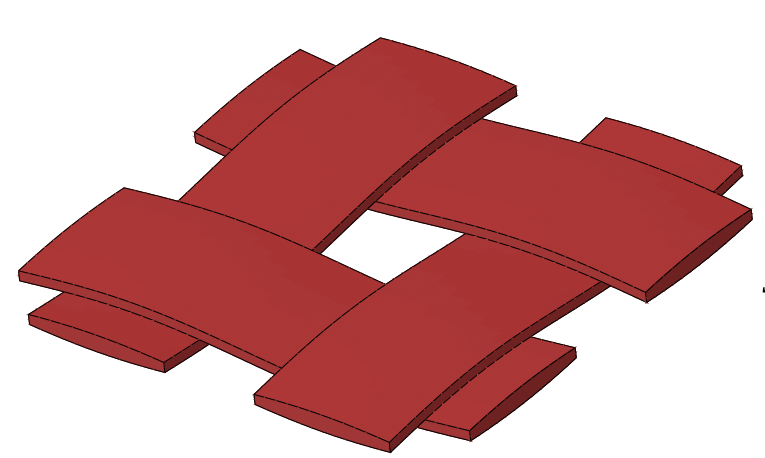

(a)

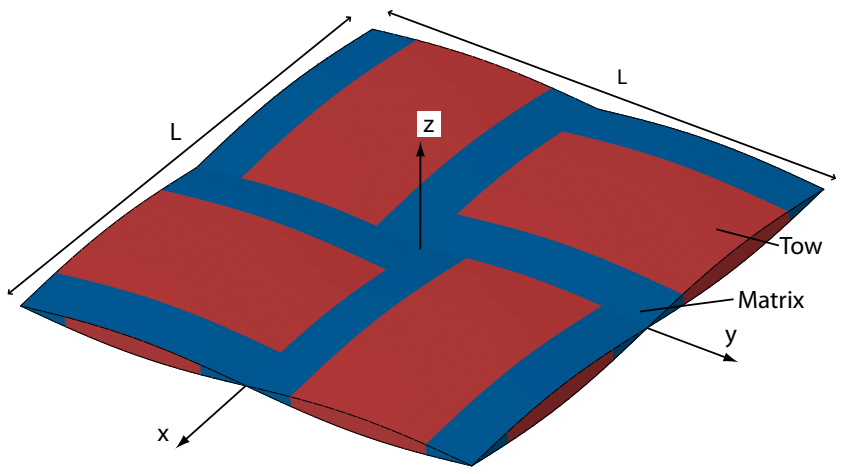

(b)

Figure 12: Finite element model for a plain-weave lamina unit cell: (a) fiber tows only, and (b) fiber tows filled with matrix.

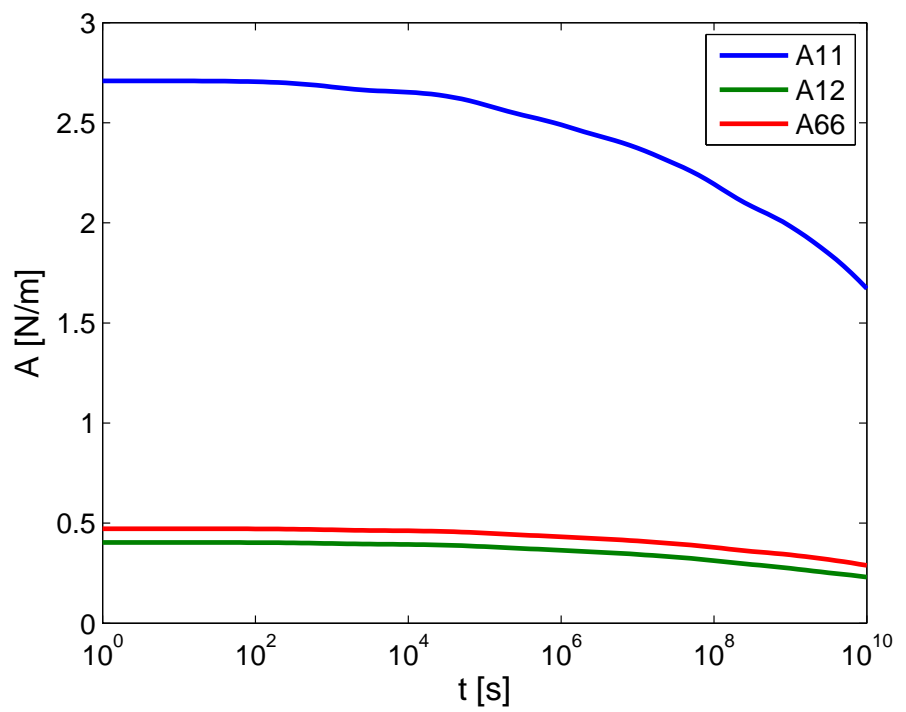

Figure 13: Viscoelastic in-plane stiffnesses of a plain-weave lamina. 


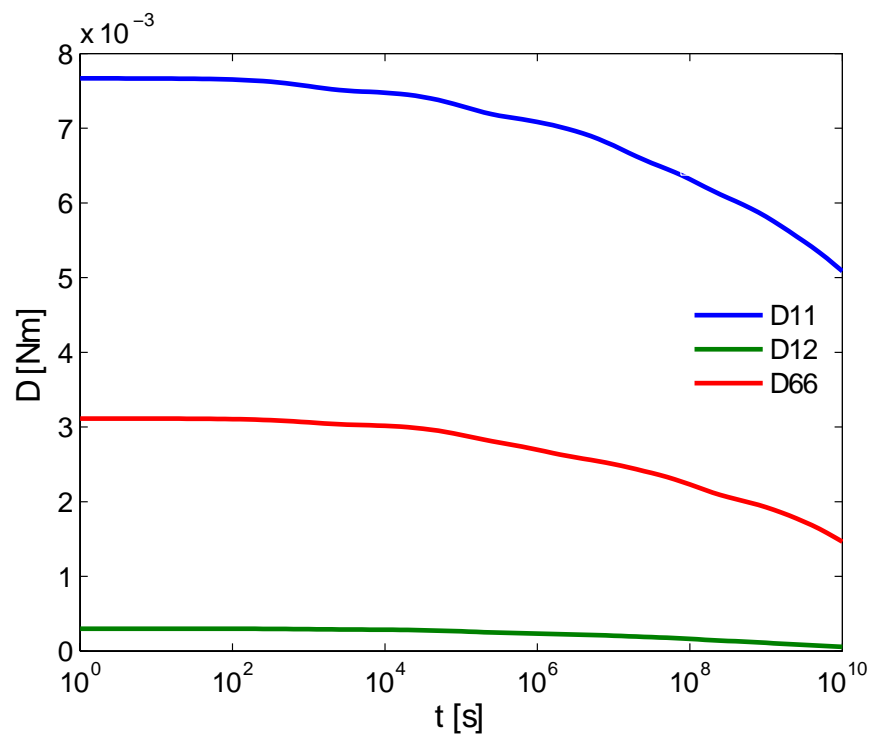

Figure 14: Viscoelastic bending stiffnesses of a plain-weave lamina.

\section{Finite Element Analysis of Tape-springs}

The deployment and recovery responses of composite tape-springs, studied experimentally in Sec. II, were simulated in Abaqus/Standard using a model with 2268 quadrilateral shell elements (S4) with a maximum dimension of $3.85 \mathrm{~mm}$. The viscoelastic stiffness properties of the shell elements were defined by assigning the $A B D$ matrix obtained in Sec. $\mathrm{C}$ via user defined shell section subroutine (UGENS). The temperature shift factor of the shell elements was taken to be the same as the that of the matrix.

The boundary conditions were applied as follows. The bottom end section was held fixed throughout the analysis. To reach the stowed configuration, the cross section at the fold region was first flattened by applying equal and opposite rotation on the two edge nodes. At the same time, a rotation of $90^{\circ}$ was applied to the middle node of the top cross section of the tape-spring. The prescribed rotations on the edge nodes were then removed and the tape-spring was kept at the stowed configuration for $8 \mathrm{~h}$. The boundary condition on the top node was released instantaneously at the end of the stowage step. The analysis was quasi-static for the folding and stowage steps, and was switched to dynamic after the removal of the top node constraint. The deployment process was simulated for $8 \mathrm{~h}$. Gravity loading was imposed throughout the simulation.

\section{Results Comparison}

Fig. 15 compares the deployed angle vs. time profile during deployment between experimental measurements and finite element simulations for $T=60^{\circ}$. Overall, the simulations show good agreement with the observed response. The overshoot angle is overpredicted by about $1.5^{\circ}$. The predicted response also shows oscillations in deployed angle. This is due to the relative large time steps used in the present simulations to reduce computational time. Nonetheless, the predicted and measured mean response are in close agreement. The measured and predicted deformed shapes of the fold during long-term recovery are compared in Fig. 16, which shows a reasonable match between the out-of-plane displacement distribution.

\section{Conclusions}

The present paper is the first systematic experimental and finite element modeling study for deployment and shape recovery behavior of thin composite space structures after stowage. It has been found that a tape-spring deploys quickly and overshoots the deployed configuration by a small amount, and then moves slowly towards its final reference configuration. The full shape recovery from the overshoot position to the fully deployed state takes place in an asymptotic manner. Stowing a composite tape-spring has the effect of 


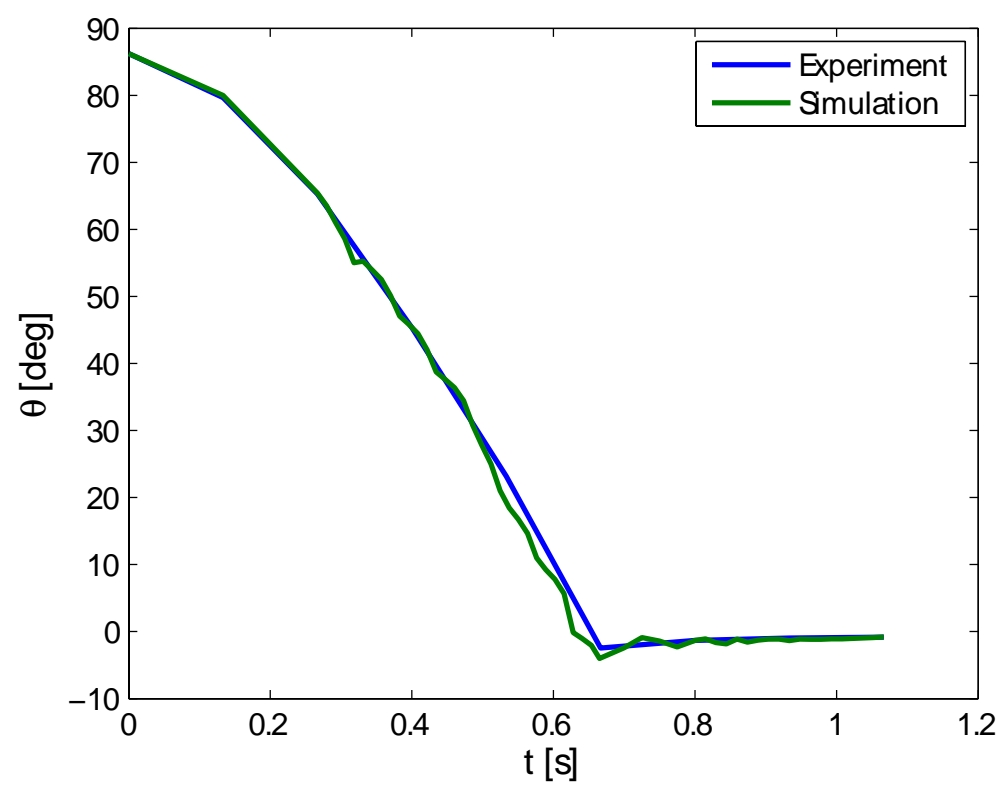

Figure 15: Comparison of deployed angle vs. time.
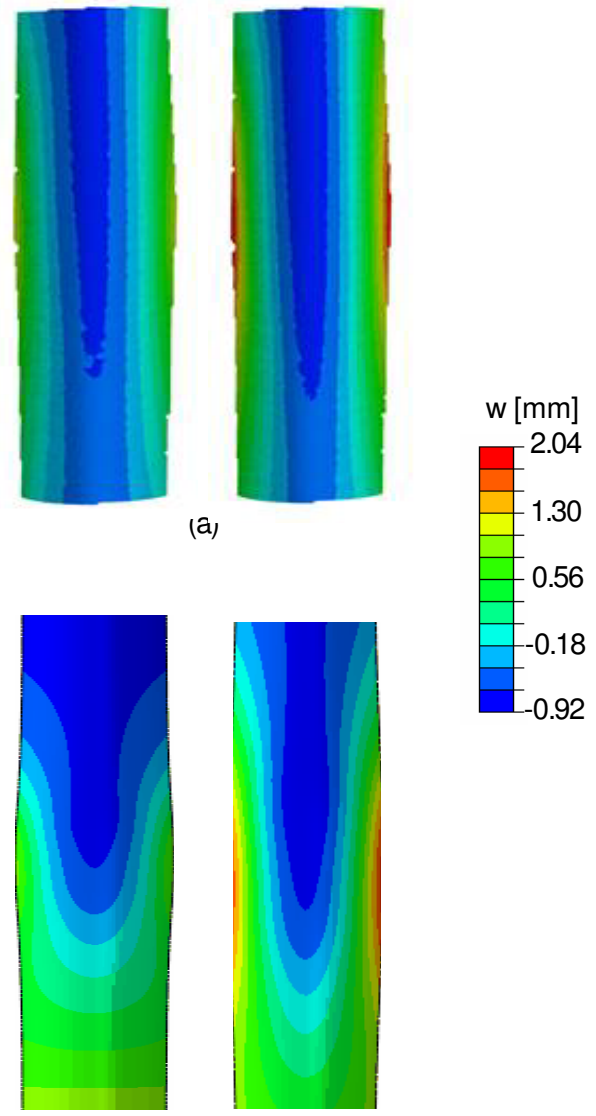

(a)

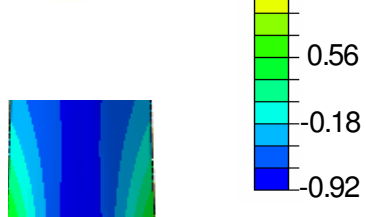

(b)

Figure 16: Comparison of recovery of fold region: (a) out-of-plane displacements measured $5 \mathrm{~s}$ after overshoot (left) and $35600 \mathrm{~s}$ after overshoot (right), (b) predicted displacements $5 \mathrm{~s}$ after overshoot (left) and $35600 \mathrm{~s}$ after overshoot (right). 
extending the time required for deployment and shape recovery.

A micromechanical finite element modeling framework for determining the homogenized viscoelastic properties of woven composite laminas has been demonstrated to be capable of capturing the temperature and time dependent behavior of thin composite structures. Both the short-term deployment behavior and the long term recovery of the localized fold are well predicted by simulations using the developed micromechanical model.

\section{Acknowledgment}

The authors are grateful to Professor Wolfgang Knauss at the California Institute of Technology for helpful comments and Gary Patz at Patz Materials Technologies for providing materials used for the present study. Financial support for Kawai Kwok by the Croucher Foundation is acknowledged.

\section{References}

${ }^{1}$ Seizt, P., "Spar resolving spat over antenna work," Space News, September 4, 1994.

${ }^{2}$ Mobrem, M. and Adams, D., "Deployment analysis of lenticular jointed antennas onboard the Mars Express Spacecraft," Journal of Spacecraft and Rockets, Vol. 46, 2009, pp. 394-402.

${ }^{3}$ Domber, J., Hinkle, J., Peterson, L., and Warren, P., "Dimensional repeatability of an elastically folded composite hinge for deployed spacecraft optics," Journal of Spacecraft and Rockets, Vol. 39, 2002, pp. 646-652.

${ }^{4}$ Soykasap, O., "Deployment analysis of a self-deployable composite boom," Composite Structures, Vol. 89, 2009, pp. 374381.

${ }^{5}$ Kwok, K. and Pellegrino, S., "Shape recovery of viscoelastic deployable structures," 51st AIAA/ASME/ASCE/AHS/ASC Structures, Structural Dynamics, and Materials Conference, No. AIAA-2010-, Orlando, Florida, 2010.

${ }^{6}$ Kwok, K. and Pellegrino, S., "Viscoelastic effects in tape-springs," 52nd AIAA/ASME/ASCE/AHS/ASC Structures, Structural Dynamics, and Materials Conference, No. AIAA-2010-, Denver, Colorado, 2011.

${ }^{7}$ Hashin, Z., "Viscoelastic behavior of heterogeneous media," Journal of Applied Mechanics, Vol. 32E, 1965, pp. 630-636.

${ }^{8}$ Hashin, Z., "Viscoelastic fiber reinforced materials," AIAA Journal, 1966.

${ }^{9}$ Brinson, L. and Knauss, W., "Finite element analysis of multiphase viscoelastic solids," Journal of Applied Mechanics, Vol. 59, 1992, pp. 730-737.

${ }^{10}$ Govindarajan, S., Langrana, N., and Weng, G., "An experimental and theoretical study of creep of a graphite/epoxy woven composite," Polymer Composites, Vol. 17, 1996, pp. 353-361.

${ }^{11}$ Shrotriya, P. and Sottos, N., "Viscoelastic response of woven composite substrates," Composite Science and Technology, Vol. 65, 2005, pp. 621-634.

${ }^{12}$ Zhu, Q., Shrotriya, P., Geubelle, P., and Sottos, N., "Viscoelastic response of a woven composite substrate for multilayer circuit board applications," Composite Science and Technology, Vol. 46, 2003, pp. 394-402.

${ }^{13}$ Seffen, K. and Pellegrino, S., "Deployment dynamics of tape springs," Proceedings of the Royal Society of London, Seris A: Mathematical and Physical Sciences, Vol. 455, 1999, pp. 1003-1048.

${ }^{14}$ Ferry, J., Viscoelastic Properties of Polymers, John Wiley and Sons, New York, 3rd ed., 1980.

${ }^{15}$ Williams, M., Landel, R., and Ferry, J., "The temperature dependence of relaxation mechanisms of amorphous polymers and other glass-forming liquids," Journal of the American Chemical Society, Vol. 77, 1955, pp. 3701-3707. 